\title{
Detection of carpal tunnel syndrome by infrared thermography
}

\author{
X. MAXEL***, J-L BODNAR**, T MARIN***, L. STUBBE* \\ * Ecole Supérieure d'Ostéopathie - Département Recherche - Cité Descartes - 8, rue Alfred Nobel - 77420 \\ Champs sur Marne, xuan.maxel@gmail.com \\ ** GRESPI/ECATHERM UFR Sciences Exactes et Naturelles Campus du Moulin de la Housse - BP 1039 \\ 51687 Reims cedex 2, jl.bodnar@univ-reims.fr
}

\begin{abstract}
The aim of this study is to approach the possibilities of stimulated infrared thermography to the carpal tunnel syndrome diagnosis. Eleven patients were studied. Six of them were affected by the carpal tunnel syndrome. The five others were healthy. The study consists in exciting the patients' hands and in analyzing their thermal response. The infrared thermograms obtained show that the hands infected by the carpal tunnel syndrome give a different thermal response.
\end{abstract}

Keywords: Carpal tunnel Syndrome, diagnosis, Stimulated infrared thermography, non-destructive testing, median nerve

\section{Introduction}

The carpal tunnel syndrome is the result of the median nerve compression [1]. At first, the pain from carpal tunnel syndrome comes with a loss of feelings in the first three fingers (thumb, index finger, and the middle finger). Actually the front of those three fingers corresponds to the median nerve innervation.

The syndrome often settles in a progressive manner. The first signs appear at night and during manual works, but they easily go away by gently hand shaking. As for the loss of feeling, it can be clinically reproduced by tests as Tinel's, Phalen's and Phalen reverse [2-5]. The evolution of the pathology makes those signs permanent. Moreover, those manifestations can be strengthened by a difficulty to hold objects; this means that the motor region of the nerve is affected. In this case, an electromyogram is often prescribed because it should bring a medico-legal evidence to attempt a surgery. But this is an invasive technique and appears to give no additional reliable diagnosis [6-8]. In the statistics of the professional troubles entitled to compensation, the carpal tunnel syndrome is the most frequent with a rate of $37 \%$ in 2002 for occupational disease referenced [9-10]. Even if in 50\% of the case, the syndrome is idiopathic, some of the traumatic and micro-traumatic causes can be caused by professional attitudes such as typing, for a long time, repeated fingers movements and the usual use of vibrating tools [11]. As the objectification of the carpal tunnel syndrome by electromyogram is invasive and no more trustworthy than usual clinical tests, it seems interesting to explore other potential diagnosis ways. The infrared thermography is a non-destructive testing already widely used to support medical diagnosis [12-23]. With this in mind, the aim of this tool is to detect any human body thermic changes. For a carpal tunnel syndrome, a lot of experimentation prove that the sympathetic nervous system quota related to the median nerve is the first to be injured [24-25]. It creates vasomotor troubles in the hand and so, potentially, local thermic variations. It seems then that using infrared thermography is a good candidate to analyze these variations [26-33]. Furthermore, the carpal tunnel syndrome first injures the non myelinated nerve fibers and this type of fiber cannot be detected by an electromyogram, which can only find the myelin sheath of the neurons [34]. This means that the infrared thermography should be able to detect the syndrome earlier. To finish, this control method is non-destructive and analysis allows remote-control without contact. For all those reasons we thought it was interesting to explore the possibilities towards detecting carpal tunnel syndrome given by the infrared thermography.

Our presentation is composed of four parts, to begin with, we will explain the way we recruited the patients, then we will expose the experimental protocol we followed, next we will present the results. Finlay, we will draw conclusions. 


\section{Experimental conditions required}

\subsection{Choice and recruitment of patient cohort who participated in the study}

We contacted health professionals (neurologist, generalists, and rheumatologists from the county and from the Osteopathic Clinique of the "Ecole Supérieure d'Ostéopathie") that could encounter people suffering from a carpal tunnel syndrome. First of all we informed them with a written paper about the goal of the research and the way the study would take place. Then, we gave them a document to inform their patient that would be susceptible to correspond to our need. At the term of this first phase, which lasted for a month, six pathological patients contacted us.

All patients met the selection criteria that we set. These are: The carpal tunnel syndrome should be diagnosed by electromyography (EMG).Patients should not have mobility problem, perform normal activities, not suffer from anxiety, depression and have no social problem. Patients had to have no pathology associated with carpal tunnel syndrome to analyze the disease only. Patient shouldn't take drug treatment. Patients could not have peripheral nervous system medication (vasodilator, antihypertensive) in order not to disrupt the sympathetic vasoconstrictor response and thus again the human body temperature medication. Patients could not be under osteopathic treatment as leading again, by disruption of the central and peripheral nervous systems, to a change in body temperature. Patients could not be in menstruation process. Patients could not participate at the time of analysis to another biomedical research, so as not to cause interference and false diagnoses. Finally, patients had to agree to comply with the study constraints. At the end of the recruitment phase, we selected eleven patients. Of these, six patients met our selection criteria and had a carpal tunnel syndrome diagnosed by electromyography. The other five were healthy subjects, showing no entrapment.

\subsection{Preliminary constraints imposed to patients.}

Once recruited, a list of 10 recommendations were given to the patient to avoid any bias by external factors such as exciting consumption, type of clothing or product application on the study area.

\subsection{Experimental protocol followed}

\subsubsection{Environmental conditions selected for the study}

The experimental protocol we followed is the one usually used for medical analysis by infrared thermography [12-21]. First of all, to avoid vasomotion phenomena we set the temperature of the room at $22^{\circ} \mathrm{C}\left(+/-1^{\circ} \mathrm{C}\right)$. In fact, the temperature of the examination room is an important element for influencing the state vascularization of skin [22]. We ensured that the experimental area was subjected to a low air exchange. We verified that humidity was maintained to a comfort zone that is to say between $40 \%$ and $70 \%$. In our study, it was typically around $50 \%$. We also sought to develop our analysis in part of sufficient dimensions, allowing some thermal stability. In our case study, these were about $10 \mathrm{~m}$ * $8 m$ * $2.5 \mathrm{~m}$. Finally, for patients' comfort and therefore to avoid generating disruptive thermal stress, we put a comfortable chair placed beside the analysis table on which the patient sat with both dorsal side hands on the table.

\subsubsection{Choice and implementation of infrared thermography camera}

At this point of the study, we had to choose the type of camera using infrared thermography and equipment to associate. To do this, we first considered the radiation properties of human skin [35]. A literature study shows that human skin has a maximum emissivity in a wavelength band between $7 \mu \mathrm{m}$ and $10 \mu \mathrm{m}$. This value is approximately 0.95 . As also the temperature of the scanned object is close to room temperature, it is therefore natural to a thermography camera "long waves" that we chose. In addition, the thermal phenomenon being studied rather slowly, it seemed sufficient to implement a bolometer array camera. So this is a camera-type A320 series27 that was used for this study.

This infrared thermography camera was mounted on a tripod of studio, to allow easy adjustment and reproducibility tests. The distance camera - hand was fixed at $90 \mathrm{~cm}$. Earlier in the day and to have images of radiometric quality, we took care to put on the camera thirty minutes before the first study. Thus, during the study the camera was in thermal equilibrium with its environment. Regarding the area taken, we standardized as done for example in radiology. It was attached to a square area of $50 \mathrm{~cm}$ side. The camera position was chosen to take advantage of the isotropic 
emission of the skin. So we set the camera perpendicularly to the analyzed hand. The support on which hands were placed was chosen polymethyl methacrylate (Plexiglas). Indeed, this material is low effusive thermally, so it will not disrupt the low temperature distribution in the hands analyzed. Moreover, it also provides a solid background that allows better reading thermograms.

\subsubsection{Patient management}

The progress of the experiment was as follows: First the patient was invited to read the experimental protocol, and then to sign in duplicate. This step was to build confidence and thus reduce temperature changes potentially generated by stress. It also aimed to verify that the patient had not consumed stimulant (coffee, tea, soda) or smoked tobacco within two hours before the experiment. He was then invited to wash his hands with soap with a neutral $\mathrm{pH}$. The objective of this phase was to remove a potential greasy film covering the hands, which can lead to local emissivity variations. The hands were then wiped with paper towels. Obviously, the patient was asked after this step not to touch anything. The patient was then asked to sit in the analysis room, positioning his hands on the analysis table, dorsal side on the table on a plate of polymethylmethacrylate, on which markers were placed to help an accurate and reproducible positioning of the hands. The table height was adjusted to the patient so that it was comfortable. This was to prevent an additional stress and a possible back pain onset. The height of the camera was then adjusted to meet the distance camera - hand defined by the protocol. Finally, the thermography recording could take place. Once the previously mentioned precautions taken, the recording could begin. The experimental protocol was then as follow: immediately after washing and drying hands, the patient was asked to place his hands on the plate of polymethylmethacrylate. To facilitate the correct positioning of the latter, reference points were marked on the plate. This initial phase was a learning stage aimed to learn the actions to be followed in the analysis. A first thermogram named to was acquired at this point. This phase, as showing the progress of the analysis, was also intended to reassure the patient. Then, the patient was asked to place the palmar surface of both hands on ice blocks for 60 seconds. This analysis phase aimed to cause sympathetic nerve stimulation. This type of excitation has been used by Nabel et al [36] to study coronary arteries vasomotion. It seemed to us a possible use for this study. After this stimulation phase the patient was asked to put his hands on the analysis table. A succession of taking infrared views was then developed. These were spaced about 30 seconds. The first is denoted $\mathrm{t} 1$ in the remainder of this article, the second $\mathrm{t} 2$ and so on until t22 to analyze the longest.

\section{Experimental results}

At first, we studied a cohort of healthy subjects. They were five. We present a patient representative of this group. Eight thermograms were performed in this patient. Contact with the ice blocks generated a temperature decrease of the hand in contact with ice. They then showed a gradual warming of these areas, but still symmetrical for both hands. Figure 1 shows the thermogram at $180 \mathrm{~s}$ after contact with the ice blocks. It shows rather symmetric thermal signatures. For both hands hypothenar areas are hotter than thenar areas. There was no more significant thermal evolution after the thermogram.

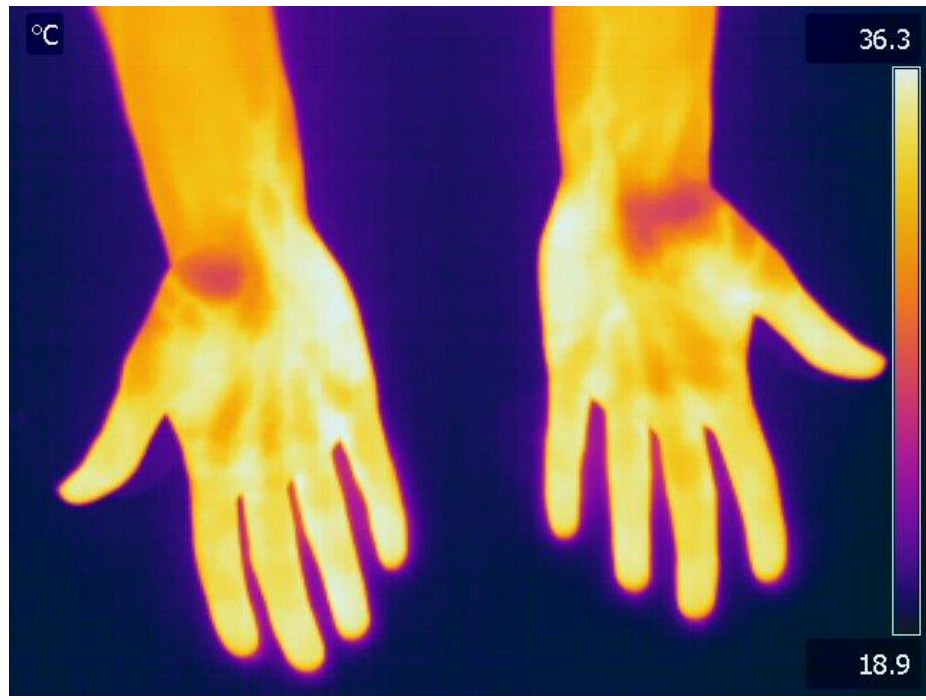

Fig. 1. Thermogram obtain during the study of healthy patient $180 \mathrm{~s}$ after contact with ice block. 
At 240 seconds after contact with the ice blocks all healthy patient show clearly a symmetrical temperature distribution on both hands.

The second part of study involved a cohort of patients with carpal tunnel syndrome. They were six. Figure 2 present an example of results obtained in this framework. The analyzed patient had carpal tunnel syndrome of his right hand. He told us suffer from nocturnal paresthesia in the thumb and forefinger of the right hand. The initial thermogram as shown in the case of a healthy subject, a symmetrical temperature distribution with both hands. Then thermal excitation revealed again as previously colder in areas where thermal contact. Finally, we found that heating the tip of the thumb and forefinger of the right hand is quicker than the other fingers. This result is interesting because it suggests as expected, that the presence of carpal tunnel syndrome can lead to disturbed vasomotion fingers affected by this disease.

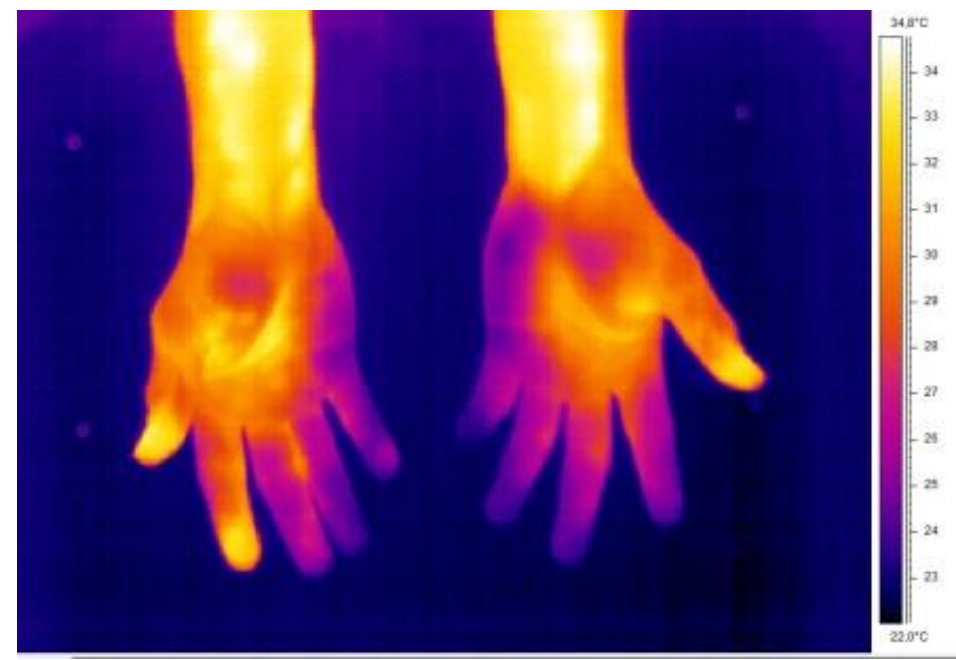

Fig.2. Thermogram obtained during the study of a subject diagnosed with carpal tunnel syndrome only on the two first fingers of the right hand 240 s after contact with ice blocks.

After we had those results from the study of a patient with carpal tunnel syndrome located on two fingers, because of the median nerve anatomical variation, we wanted a second case presented syndrome having another anatomical variation. This is certainly the most common and affects the first three fingers (thumb, index, middle). As before, we noted an initial image showing a thermal symmetry with both hands. After contact with the ice blocks however it shows a different thermal behavior of the right hand three fingers with the carpal tunnel syndrome. Figure 3 shows the particular behavior after 90 seconds contact (thermal abnormal behavior on left ring. However, it is probably due to the presence of an alliance disrupting vasculature finger is noted). This result confirms the previous one. It also shows that the infrared technique is sensitive to the median nerve anatomical variation.

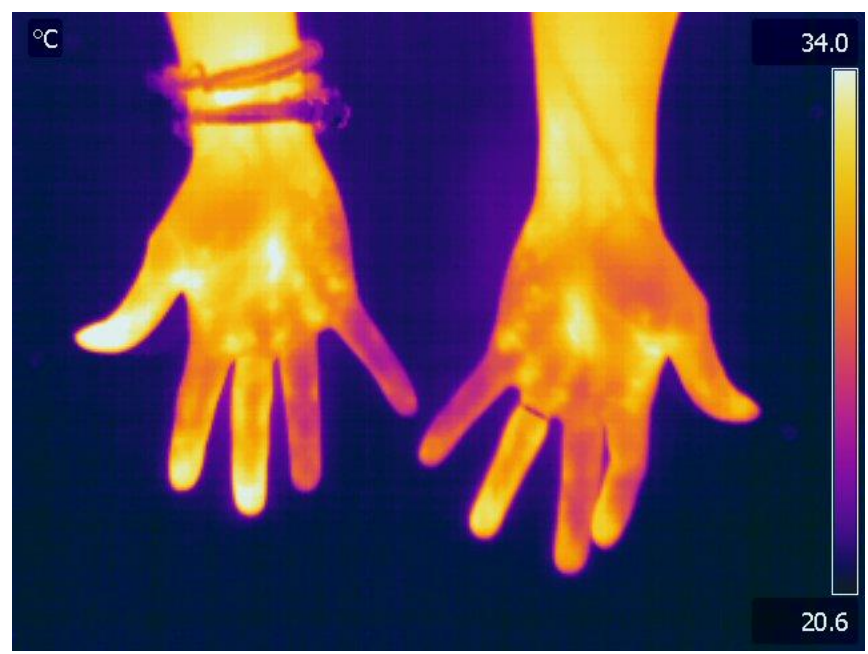

Fig. 3. Thermogram obtained during the study of a subject diagnosed with carpal tunnel syndrome in three fingers of the right hand, at $90 \mathrm{~s}$ after contact with ice blocks. 
At last, we had to determine criteria to differentiate the patient suffering from the carpal tunnel syndrome from the healthy one. With that in mind, we first had to calculate the hands' reference temperature. This temperature is the one equal to the temperature of the thumb and the index, from which we subtract the temperature of the ring and last fingers. In the case of a healthy hand, the reference temperature is approximately $0{ }^{\circ} \mathrm{C}$ and quite homogeneous. If the patient presents a carpal tunnel syndrome, the difference increases because the thermic comportment of the hand is different. Table 10 is a table where we show the results for the eleven patients we had. We can see that the variation of the temperature is weak for healthy patients and increases for pathological patients. It also proves that the variation of temperature should be above or under $0^{\circ} \mathrm{C}$. It corresponds to a vasoconstriction or vasodilatation of the veins.

\begin{tabular}{|c|c|c|}
\hline Patient & $\Delta \mathrm{T}$ right hand & $\Delta \mathrm{T}$ left hand \\
\hline Healthy 1 & 0,9 & $-0,4$ \\
\hline Healthy 2 & $-0,8$ & $-0,1$ \\
\hline Healthy 3 & 0,6 & 0,2 \\
\hline Healthy 4 & $-0,1$ & 0 \\
\hline Healthy 5 & 0 & $-1,5$ \\
\hline CTS 1 right & 16,2 & 0 \\
\hline CTS 2 right & 3,6 & 0,8 \\
\hline CTS 3 right & 13,3 & 0,1 \\
\hline CTS 4 right & 9,3 & 1,8 \\
\hline CTS 5 bilateral & 7,8 & 13,9 \\
\hline CTS 6 bilateral & 3,8 & $-12,6$ \\
\hline
\end{tabular}

Table 1. Difference in characteristic measured temperature in both hands healthy and diseased patients studied. 


\section{Conclusion}

In this work, we approached the possibilities of stimulated infrared thermography for carpal tunnel syndrome detection. We first reported, following a literature review the causes of arterial vasomotion at hand. Indeed, the sympathetic quota led by the median nerve has no myelin sheath, making them more susceptible to compression. It then modifies the threshold of excitability of nerve fibers based on these phenomena and therefore bloodstream. But a change in the circulation can cause a variation of the hand temperature, which is then detected by infrared thermography. Moreover, the sensory nerve fibers are surrounded by a myelin sheath; they will be affected later than the sympathetic fibers. Infrared thermography could allow early detection of the disease carpal tunnel. Moreover, the conventional method (EMG) is recognized $82 \%$ reliable only. As also it is invasive, it is worth trying to find a way of non-invasive diagnosis and why not more reliable. Thermography can in fact be a candidate. We then proposed an experimental protocol to be more rigorous and repeatable as possible. We explained the choice of a long wave infrared thermography camera. In the fourth step, we showed that the fingers with a carpal tunnel syndrome had a different thermal behavior, which enables the detection of the disease. At this stage, we showed also that the method allowed detection of anatomical variation on the distribution of the median nerve. In the fifth step, we proposed a quantification of the detection method. To do so, we defined a characteristic temperature variation and showed that it was close to zero for healthy subjects and higher for pathological subjects. This study of a cohort of restricted patient now needs to be generalized. Moreover, the robustness of the latter should be studied. It would be interesting to understand why the thermal signature of the carpal tunnel syndrome was generally positive but also negative in a particular case. It would also be interesting to investigate the cause of the kinetics of thermal reactions different from one patient to another. It would still be necessary to optimize the experimental protocol (duration excitement, excitement type, duration analysis, type of post-processing ...). Finally, the influence and pertinence of selected exclusion criteria during this study should be considered as well. Studies in this direction are underway.

\section{REFERENCES}

[1] Kernbaum S. Dictionnaire de médecine flammarion. Paris : Flammarion Médecine-Sciences;2008.

[2] El Miedany Y, Ashour S, Youssef S, Mehanna A, Meky F. Clinical diagnosis of carpal tunnel syndrome: Old testsnew concepts, Joint Bone Spine, Volume 75, Issue 4, July 2008, Pages 451-457, ISSN 1297-319X, 10.1016/j.jbspin.2007.09.014.

[3] Durkan J. A new diagnostic test for carpal tunnel syndrome. J Bone Joint Surg Am 1991;13:535-8

[4] Tinel J. Le signe du « fourmillement » dans les lesions des nerfs périphériques. Press Med $1915 ; 23: 388$.

[5] Phalen GS. Reflections on 21 year'experience with the carpal tunnel syndrome. JAMA $1970 ; 212: 1365-7$.

[6] Szabo RM, Slater Jr RR, Farver TB, Breger Stanton D, K. Sharman WK. The Value of Diagnostic Testing in Carpal Tunnel Syndrome, The Journal of Hand Surgery, Volume 24, Issue 4, 1999, Pages 704-714, ISSN 0363 5023, 10.1053/jhsu.1999.0704.

[7] Redmond MD, Rivner MH. False positive electrodiagnostic tests in carpal tunnel syndrome Muscle Nerve, 11 (1988), pp. 511-518.

[8] J.C. White, S.R. Hansen, R.K. Johnson, A comparison of EMG procedures in the carpal tunnel syndrome with clinical-EMG correlations Muscle Nerve, 11 (1988), pp. 1177-1182.

[9] Haute Autorité de Santé. Chirurgie du syndrome du canal carpien: approche multidimensionnelle pour une décision pertinente. Décembre 2011.

[10] Institut National de la Recherche Scientifique. Régime général Tableau 57 [document électronique] http://www.inrs-mp.fr. Dernière mise à jour 17/10/2011.

[11] Collège Français des Enseignants en Rhumatologie. Précis de rhumatologie. Paris : Masson ;2002.

[12] Baillie AJ, Biagioni PA, Forysth A, Garioch JJ, and McPherson D. Thermographic assessment of patch-test responses. Br J Dermatol 122: 351-360, 1990.

[13] Bharara M, Cobb JE, and Claremont DJ. Thermography and thermometry in the assessment of diabetic neuropathic foot: a case for furthering the role of thermal techniques. Int J Low Extrem Wounds 5: 250-260, 2006.

[14] Bitar D, Goubar A and Desenclos JC. International travels and fever screeining during epidemics : a literature review on the effectiveness and potential use of non-contact infrared thermometers. Eurosurveillance, 14 (6) : 1 5.

[15] Buzug TM, Schumann S, Pfaffmann L, Reinhold U, and Ruhlmann J. Functional Infrared Imaging for SkinCancer Screening. Conf Proc IEEE Eng Med Biol Soc 1: 2766-2769, 2006.

[16] Forestier J, Kuerth A, Niederstrasser E,Krautwald E, Pauli R, Paulat R, Eweleit M, Riemekasten G, and Worm M. A cold-response index for the assessment of Raynaud's phenomenon. J Dermatol Sci 45: 113-120, 2007.

[17] Hartmann M, Kunze J, and Friedel S. Telethermography in the diagnostics and management of malignant melanomas. J Dermatol Surg Oncol 7: 213-218, 1981.

[18] Head JF and Elliott RL. Infrared imaging: making progress in fulfilling its medical promise. IEEE Eng Med Biol Mag 21: 80-85, 2002. 
[19] Renkielska A, Nowakowoski A, Kaczmarek M and Ruminski J. Burn depths evaluation based on active dynamic IR thermal imaging--a preliminary study. Burns 32: 867-875, 2006.

[20] Ring EF, Engel JM, and Page-Thomas DP. Thermologic methods in clinical pharmacology-skin temperature measurement in drug trials. Int J Clin Pharmacol Ther Toxicol 22: 20-24, 1984.

[21] Siniewicz K, Wiecek B, Baszczynski J, Zwolenik S. The importance of thermovision in children with orthostatic cardiovascular disorders before and after directed physical exercises, a casebook of infrared imaging in clinical medicine, medpress 2003, pp 87 - 93

[22] Ming Z, Zaproudina N, Siivola J, Nousiainen U, Pietikainen S. Sympathetic pathology evidenced by hand thermal anomalies in carpal tunnel syndrome, Pathophysiology, Volume 12, Issue 2, September 2005, Pages 137-141, ISSN 0928-4680, 10.1016/j.pathophys.2005.05.002.

[23] Tchou S, Costich JF, Burgess RC, Wexler CE. Thermographic observations in unilateral carpal tunnel syndrome: Report of 61 cases, The Journal of Hand Surgery, Volume 17, Issue 4, July 1992, Pages 631-637, ISSN 03635023, 10.1016/0363-5023(92)90306-A.

[24] Ming Z, Siivola J, Pietikainen S, Närhi M, Hänninen O. Postoperative relieve of abnormal vasoregulation in carpal tunnel syndrome, Clinical Neurology and Neurosurgery, Volume 109, Issue 5, June 2007, Pages 413-417, ISSN 0303-8467, 10.1016/j.clineuro.2007.02.014.

[25] Lang E, Claus D, Neundörfer B, Handwerker HO. Parameters of thick and thin nerve-fiber functions as predictors of pain in carpal tunnel syndrome, Pain, Volume 60, Issue 3, March 1995, Pages 295-302, ISSN 0304-3959, 10.1016/0304-3959(94)00131-W

[26] Özden Şener H, Taşcılar NF, Balaban H, Selçuki D. Sympathetic skin response in carpal tunnel syndrome, Clinical Neurophysiology, Volume 111, Issue 8, 1 August 2000, Pages 1395-1399, ISSN 1388-2457, 10.1016/S1388-2457(00)00333-3.

[27] Gautherie M, Jesel M, Daemgen F, Meyer S. Troubles vasomoteurs des mains et syndrome du canal carpien, Annales de Chirurgie de la Main et du Membre Supérieur, Volume 14, Issue 2, 1995, Pages 85-95, ISSN 11532424, 10.1016/S0753-9053(05)80394-1.

[28] Léger O, Lavallé F. Révélation d'un syndrome du canal carpien par ulcération digitale secondaire à un vasospasme artériel, Chirurgie de la Main, Volume 24, Issue 1, February 2005, Pages 39-41, ISSN 1297-3203, 10.1016/j.main.2004.11.007.

[29] Tortora,G.J., Grabowski, S.R. (2001). Principes d'anatomie et de physiologie. Adaptation française Andrée Imbach et André Ferron. Alternative Therapies, Janvier, Vol 2, №1.

[30] Malpas, S.C. (1998). The rhytmicity of sympathetic nerve activity. Progress in Neurobiology, Volume 56, Issue1, Septembre 1998, p.65-96.

[31] Fujii, K.,Heistad, D.D., Faraci, F.M. (1990). Vasomotion of basilar arteries in vivo. Am J Physiol., Juin, 258(6 Pt 2), p.H1829-34..

[32] Bouskela, E., Grampp, W. (1992). Spontaneous vasomotion in hamster cheek pouch arterioles in varying experimental conditions. Am J physiol., Février, 262(2 Pt2), p.H478-85,

[33] Shahani B, J-Halperin J, Boulu P. Cohen J. Sympathetic skin response-a method of assessing unmyelinated axon dysfunction in peripheral neuropathies Journal of Neurology, Neurosurgery, and Psychiatry 1984;47:536542.

[34] Guyton, A.C. (1991). Textbook of Medical Phisiology. 8ème édition. USA, Philadeplhia W.B. Saunders Company.

[35] R. Bowling Barnes. Infrared-radiant energy provides new concepts and instrumentation for medical diagnosis, Science 24 May 1963: 870-877.

[36] Nabel EJ, Ganz P, Gordon JB, Alexander RW, Selwyn AP. Dilation of normal and constriction of artherosclerotic coronary arteries caused by the cold pressor test. Circulation 1988;77:43-52. 